\title{
Non-destructive Measurement of Magnetic Properties of Claw Pole
}

\author{
Chengliang Hu${ }^{1}$, Xuejiao Bai ${ }^{1}$, Minjun Tang ${ }^{2}$, Xiaofeng Tang $^{2}$ and Zhen Zhao ${ }^{1 *}$
}

\begin{abstract}
The magnetic properties of the claw pole have a direct effect on the output power of a generator. Many methods can be used to measure these magnetic properties, each with its own advantages, but an important shortcoming is that all are destructive. In this study, a new non-destructive method to measure the magnetic properties of claw pole was proposed and a corresponding testing set-up was designed. A finite-element model was constructed to simulate the measurement process. Results proved that the measured magnetization-like curves had good agreement with the trend of the input magnetic curves and the effect of the positioning error in the measuring process could be neglected. To further validate the new method, seven types of claw poles of different materials subjected to different heat-treatment processes were forged and tested by both the new method and the conventional ring-sample method. Compared with the latter, the new method showed better consistency, relatively higher accuracy, and much stronger stability of measurement results; however, its sensitivity needs to be improved. The effects of material compositions and heat-treatment parameters on the magnetic properties of the claw pole were briefly analyzed.
\end{abstract}

Keywords: Claw pole, Magnetic property, Measurement, Ring sample, Accuracy, Stability

\section{Introduction}

The claw pole is a necessary component of a synchronous generator with direct-current (DC) excitation, which is commonly used in the automobile industry because of its low cost and ease of regulation [1]. Claw poles are mainly manufactured by hot forging: common forging schemes include closed-die forging [2], casting-forging [3], single forging with a spring-controlled die [4], and radial forging with forward extrusion [5]. After forging, claw poles are heat treated and machined for subsequent assembly.

Two claw poles cooperate with each other to form the magnetic poles of the rotor, as shown in Figure 1. They are magnetized under the excitation of the energized magnetizing winding and then generate a strong magnetic field [6]. The fundamental magnetization curve of the material is the most important curve characterizing the magnetic properties of a claw pole, reflecting its ability to be excited and magnetized under the action of an

\footnotetext{
*Correspondence: zzhao@sjtu.edu.cn

${ }^{1}$ Institute of Forming Technology \& Equipment, School of Material Science and Engineering, Shanghai Jiao Tong University,

Shanghai 200030, China

Full list of author information is available at the end of the article
}

external magnetic field. The greater the magnetic induction of the material under the same excitation current, the greater the output current of the claw pole generator, and the better the power generation performance.

Magnetic properties can be measured in a closed or open circuit. The simplest example of a closed magnetic circuit is a ring, which need not be limited to a circular cross-section [7]. It is also possible to obtain the magnetization curve and hysteresis loop using a hollow square or other closed geometrical shape [8]. The effect of precipitate element addition on the magnetic properties of the magnetostrictive $\mathrm{Fe}_{83} \mathrm{Ga}_{17}$ alloy was studied with ring samples: the results showed that the coercivity significantly increased with the addition of such elements [9]. Addition of boron caused undesirable deterioration of the magnetic properties of a permalloy, while the effects of magnesium and calcium on its magnetic properties were small [10]. A ring sample can easily form a closed magnetic circuit, but the disadvantages of this method include: (1) considering the thermal effect, it is not possible to apply very large circumferential fields [11]; (2) for a real part like a claw pole, the measured results are closely related to the sampling position; (3) it can be 


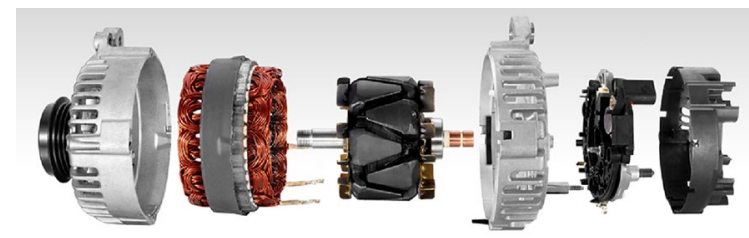

Figure 1 Claw pole and generator

time-consuming because primary and secondary windings must be applied to each sample.

Magnetic measurements in open circuit are usually performed with a vibrating-sample magnetometer (VSM) [12], alternating-gradient magnetometer [13], fluxmeter, or superconducting quantum-interference device magnetometer $[14,15]$. Of these the VSM is most popular. The magnetic flux density and coercivity of new Fe84xNb2B14Cux nanocrystalline alloys were measured with a VSM under an applied field of $8 \times 10^{5} \mathrm{~A} / \mathrm{m}$, and it was found that the magnetic properties were enhanced with appropriate $\mathrm{Cu}$ addition and annealing conditions [16]. The magnetic hysteresis loops of $\mathrm{Co} / \mathrm{CoFe} \mathrm{O}_{4}$ nanoparticles were measured using a VSM with a maximum field of $\pm 1.6 \times 10^{6} \mathrm{~A} / \mathrm{m}$, and a demagnetization jump at low fields was found [17]. A low-cost VSM was successfully constructed by using an electromagnet and an audio loud speaker, which could record the magnetic hysteresis loop up to $6.6 \times 10^{5} \mathrm{~A} / \mathrm{m}$ at room temperature [18]. The magnetic properties of Tb32Co68 after adding a [SiN $x$ / $\mathrm{Co}]_{n}$ underlayer were analyzed by VSM with a maximum applied field of $1.6 \times 10^{6} \mathrm{~A} / \mathrm{m}$, and the saturation magnetization value of the multilayer films was enhanced [19]. The ferromagnetic behavior of Ni-Zn ferrite-epoxy composite thin films was revealed by VSM, and the degree of saturation magnetization of the composites increased with the addition of NiZn ferrite nanoparticles [20]. An optimized VSM design was determined using numerical calculations, based on the principle of reciprocity and the Biot-Savart law, and was able to detect the magnetic moment of a $100 \mathrm{~nm}$-thick $\mathrm{Fe}_{19} \mathrm{Ni}_{81}$ film and its change of $\sim 2 \times 10^{-7} \mathrm{~A} \mathrm{~m}^{2}$ [21]. However, VSMs of normal design are only applicable to samples with small dimensions, thin films, or other small electronic components.

Magnetic parameters can also be measured using a permeameter [22], in which a closed magnetic circuit is formed by attaching a yoke, or yokes, of soft magnetic material to the sample. Various types of permeameters have been proposed, which differ in the size and shape of the sample, yoke, magnetizing winding, and other characteristics. A semi-rigid cable loop was proposed to work as a single-turn shielded-loop pick-up coil for a highfrequency permeameter, and it could measure permeability up to $3 \mathrm{GHz}$ with a simple structure [23]. A fully automatic system for measuring magnetic properties of soft and hard ferromagnetic materials was designed and it can adopt a variety of test configurations, including a bar permeameter, ring samples, or Epstein frame [24]. A technique was developed to calibrate the performance of a special permeameter for measuring soft magnetic materials by using cylindrical standard samples, and the true $M(H)$ curve for standard samples of large length-todiameter ratios was obtained by the demagnetizing-corrected solenoid method [25]. Because a non-measured medium exists in the magnetic circuit using a permeameter, the abscissa of the measured $B-H$ curve is not the actual external magnetic field intensity applied to the measured medium. This instrument is not suitable for evaluating the magnetic properties of a complex part.

In industry, more attention is paid to the overall magnetic properties of claw pole. If the above methods were used, the sampling process would destroy the part and the specific sampling position would directly affect the measurement. In this work, a Non-destructive Test for Evaluating the Magnetic properties of claw pole (NDTEM) was studied. In the following sections, the design concept of this new measurement method is introduced in detail. In Section 3, a finite-element (FE) model is described, which was constructed according to the concept, and an attempt to verify the correctness and effectiveness of the simulation results is made. Section 4 describes a group of experimental tests that was performed to validate the NDTEM, and specific schemes for both the NDTEM and ring-sample methods are presented. In Section 5, the consistency evaluation, accuracy analysis, and stability analysis of the two methods are discussed, and the magnetic properties of different claw poles are briefly evaluated based on the results measured by the NDTEM. Finally, the conclusions drawn from this research are summarized in Section 6.

\section{New Measurement Method}

In the test method using a ring sample, the ring itself forms a closed magnetic circuit. In the method using a permeameter, a rod sample with a yoke in the permeameter constitutes a closed circuit. In the proposed NDTEM, a claw pole to be measured, a support base, and a guide ring are used to construct a complete magnetic circuit, as shown in Figure 2. Here, the support base and guide ring were made of electric iron DT4 that has good magnetic properties to ensure that the support base, guide ring and claw pole can form a closed magnetic circuit. The positioning axis and winding ferrule were made of aluminum alloy $2 \mathrm{~A} 12$ that has a magnetic permeability of around 1 $\mathrm{H} / \mathrm{m}$, so that they have almost no influence on the magnetic circuit. The magnetic line of force, indicated by small arrows, was vertical to the end face of the boss on 


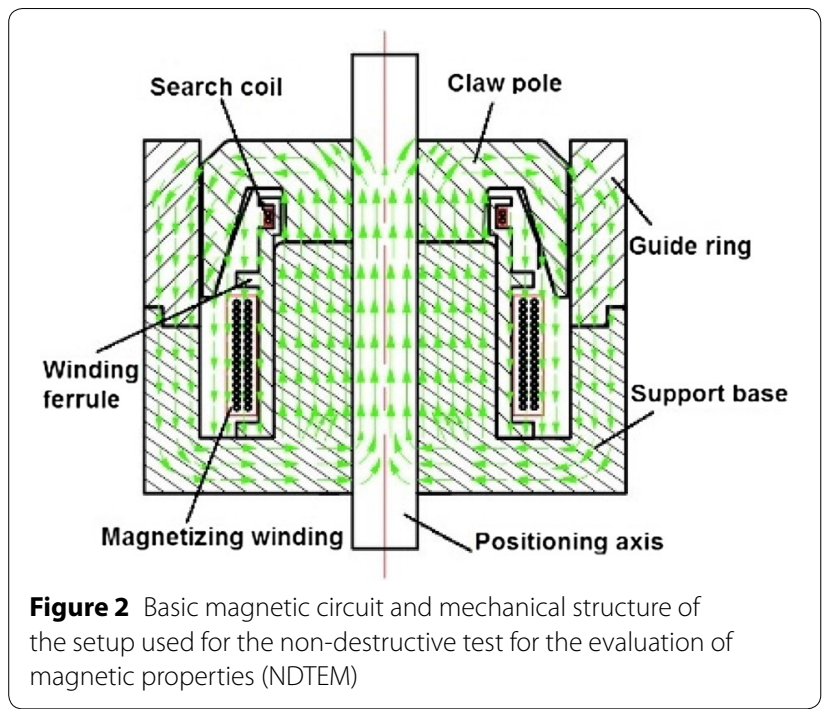

the claw pole and went through the claw part to the outer edge surface and the air, then penetrated to the guide ring. After passing over the outer ring and inner boss of the support base, the magnetic line of force entered the boss of the claw pole again, forming a closed magnetic circuit.

The designed magnetic circuit had a high degree of similarity with the magnetic circuit formed by a claw pole working in a motor. The magnetic field of a claw pole in operation goes through the air gap after passing through the claw part to the outer edge surface, enters the stator yoke, passes through the air gap again, and then penetrates the outer edge face of the adjacent claw of another paired claw pole. Therefore, the magnetic force lines are twisted between the paired claw poles. However, for each claw pole, the magnetic line of force always passes from the outer edge of the claw part and the air gap to the stator yoke.

The winding ferrule had two spaces for coils. The magnetizing winding was wound on the lower space where the inner boss of the support base was covered, and the search coil was wound on the upper space where the inner boss of the claw pole was covered. The excitation effect of the magnetizing winding was the same as that of the magnetizing winding inside the rotor of a claw pole generator. When the magnetizing winding was connected to the inrush current, a voltage and current were induced in the search coil. The relationship between the inductive voltage and time was obtained using the galvanometer. By integration, the magnetic flux passing through the cross-section of the inner boss of the claw pole was collected, and the corresponding magnetic induction intensity was calculated by division by the area of the cross-section. The curve of magnetic induction

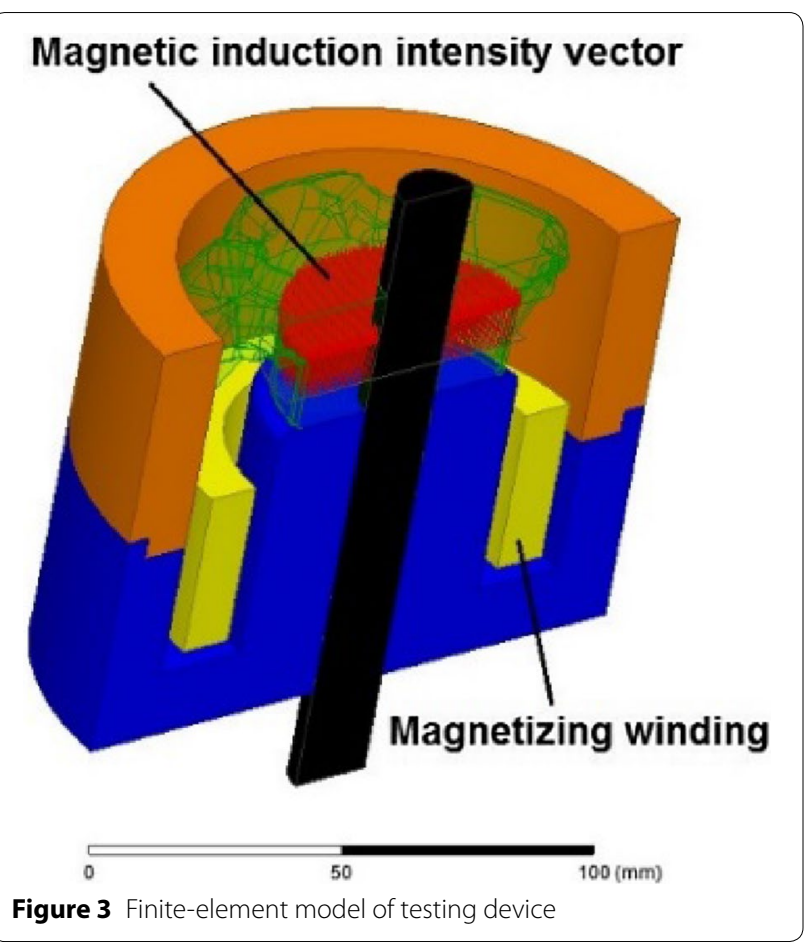

intensity in the magnetic circuit that varied with the excitation current could then be obtained, which was used to characterize the magnetic properties of the claw pole. In the process of measurement, if the same support base and guide ring were used, claw poles with the same outer diameter could be interchanged, so the magnetic properties of the same type of claw poles produced by different manufacturing processes could be evaluated. Of course, the dimensions of the support base and guide ring can be designed based on different sizes of claw poles for testing of their magnetic properties.

\section{Validation by Numerical Simulation}

To verify the correctness and effectiveness of the NDTEM, numerical analysis of the magnetic measuring process was carried out by FE simulation. To simplify the modeling process and reduce the calculation time, an equivalent static magnetic analysis model was used to solve the transient magnetic field problem for the entire measurement system.

\subsection{Finite-Element Modeling}

According to the above concept, a corresponding FE model was created in ANSYS-Maxwell, as shown in Figure 3. The main dimensions of the claw pole to be measured are illustrated in Figure 4. The maximum outer diameter and height were $98.30 \mathrm{~mm}$ and $42.20 \mathrm{~mm}$, respectively, and the magnetization curve of the raw 


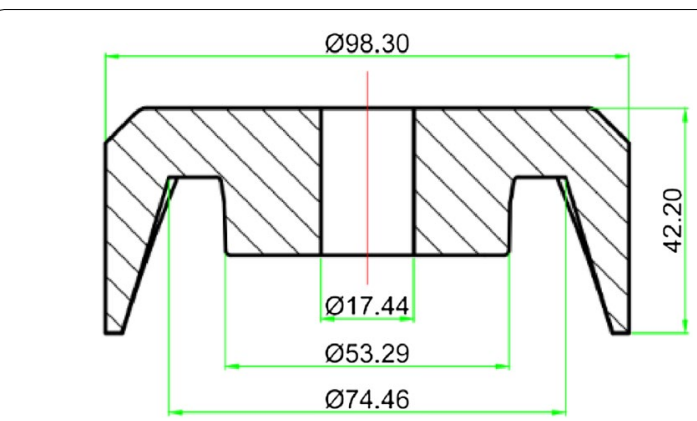

Figure 4 Specific dimensions of modeled claw pole

Table 1 Parameter values for finite-element model

\begin{tabular}{ll}
\hline Parameters & Values \\
\hline Maximum number of passes & 12 \\
Percent error & 1 \\
Refinement per pass & $30 \%$ \\
Minimum number of passes & 2 \\
Minimum converged passes & 1 \\
Nonlinear residual & 0.001 \\
Relative residual & $1 \times 10^{-6}$ \\
\hline
\end{tabular}

material of the claw pole was input. The materials data for the support base, guide ring, and positioning axis were selected from the database of the software. In the entire solution domain, the parameter of air was also defined. In the simulation, all magnetic parameters of the materials were assumed to be isotropic.

The magnetizing winding, which consisted of twisted copper conductors with a diameter of $0.8 \mathrm{~mm}$, is equivalent to a ring: it was set as a "stranded" type, in which the eddy current effect was ignored. The corresponding magnetic parameters of the copper conductor were defined using the material database. The number of magnetizing winding turns in the claw pole motor was 360; an appropriate increase in the number of turns could reduce the output current of the equipment. The number of magnetizing winding turns used for the designed measuring system was 400 , and the excitation current could be varied in the range of $0.00-3.75 \mathrm{~A}$. Adaptive analysis technology was used to mesh the tetrahedral elements for all parts. This was an iterative process to form an initial mesh with fewer elements and subdivide the mesh of some regions according to the calculated magnetic distributions. The specific parameters of the solution setup are shown in Table 1.

In the actual measurement process, the average magnetic induction intensity was obtained. In the simulation, the magnetic flux was the integration of the magnetic flux density through the cross-section of the inner boss in the claw pole, so the average magnetic induction intensity was calculated according to Eq. (1):

$$
\bar{B}=\frac{\int B \cdot \mathrm{d} s}{S}
$$

where $B$ is magnetic flux density, $S$ is the cross-sectional area of the inner boss in the claw pole, and $\bar{B}$ is the average magnetic induction intensity.

By changing the input excitation current from $0.00 \mathrm{~A}$ to $3.75 \mathrm{~A}$, a magnetization-like curve of the claw pole could be measured. The average magnetic induction intensity was selected as the ordinate, while the total current (which is the product of the excitation current and the number of turns of the magnetizing winding) was defined as the abscissa.

The relationship between the magnetization curve and the magnetization-like curve is determined by Ampere circuital theorem, as shown in Eq. (2):

$$
\oint H \cdot \mathrm{d} l=l_{e} \cdot H=\sum N I,
$$

where $H$ is the intensity of external magnetic field, $l_{e}$ is the length of equivalent magnetic circuit, $N$ is the number of turns of the magnetizing winding, $I$ is the excitation current.

So, there exists a ratio $l_{e}$ between the abscissa of the two type of measured curves. In this work, the magnetization-like curve was used to reflect the overall magnetic performance of the claw poles.

\subsection{Simulation Results}

To analyze the measurement process in detail, specific magnetization curves, which were measured using the ring-sample method, of two claw poles made by different manufacturing processes (shown in Figure 5) were imported into the constructed FE model to simulate the static magnetic field.

Figure 6 shows the simulated result of the magnetic field distribution of the measuring system when the magnetizing winding was energized. The magnetic flux density through the cross-section of the inner boss of the claw pole was quite uniform and the absolute value was larger than that of other zones.

This also proved that the use of Eq. (1) for the calculation of magnetic induction intensity was reasonable. In this case, the magnetic induction intensity obtained from the cross-section was much more representative. Therefore, the magnetic properties of claw poles could be evaluated by comparing the measured magnetization-like curves.

Figure 7 shows the magnetization-like curves obtained from simulation results. The magnetic induction 


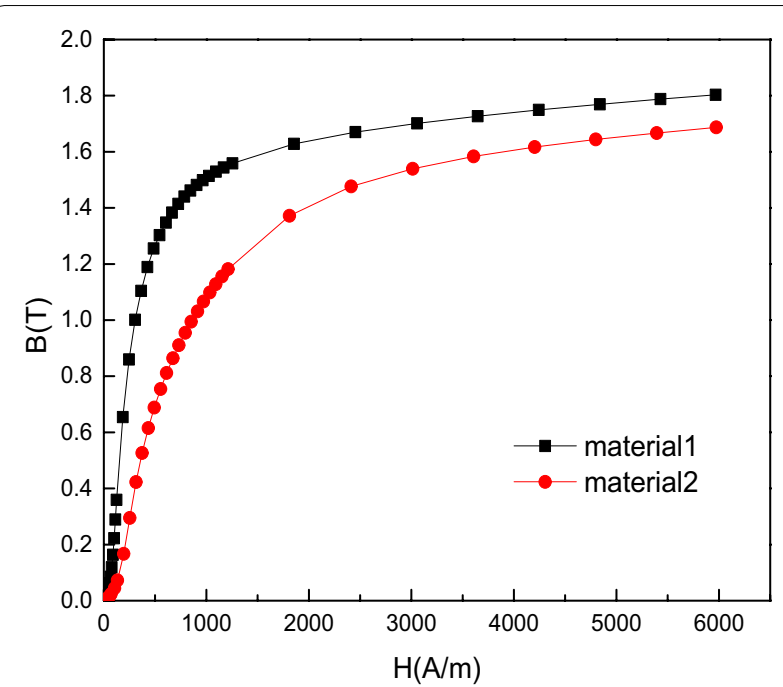

Figure 5 Magnetization curves of two different claw poles

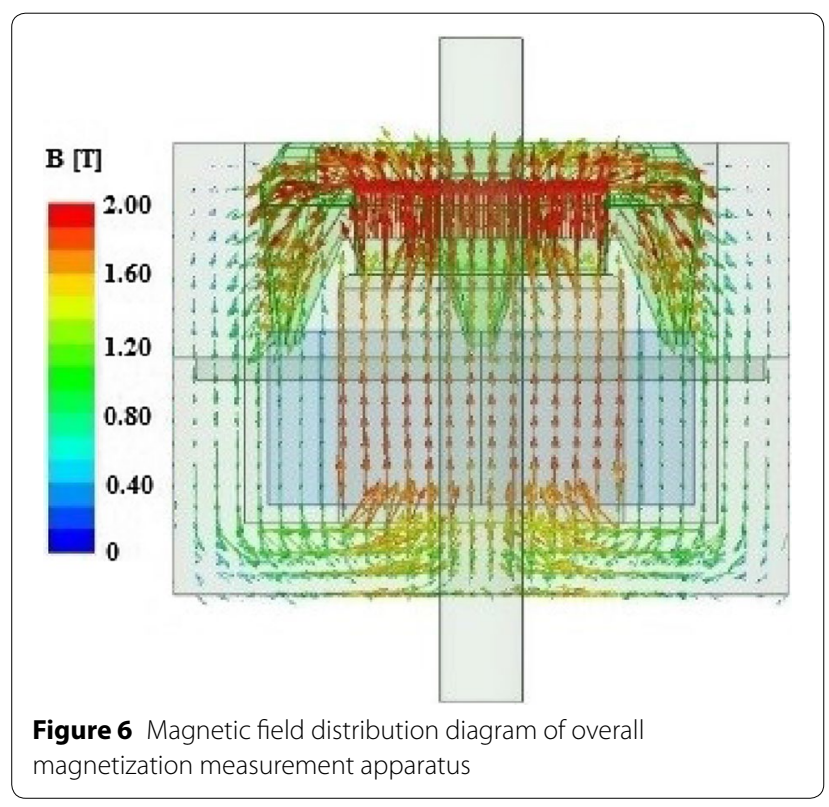

intensity of the claw poles increased with the total current, as expected. Compared with the input magnetization curves shown in Figure 5, there is good agreement in the trend of the curves, and the difference in magnetic properties of the two claw poles can be clearly identified. Therefore, the NDTEM can be used to measure the magnetic properties of claw poles.

In actual practice, the manufacturing process and installation for measurement will introduce some errors in positional deviations of the claw pole. In the simulation, the claw pole position was assumed to be offset by $0.5 \mathrm{~mm}$ in both the $X$ and $Y$ directions. These results

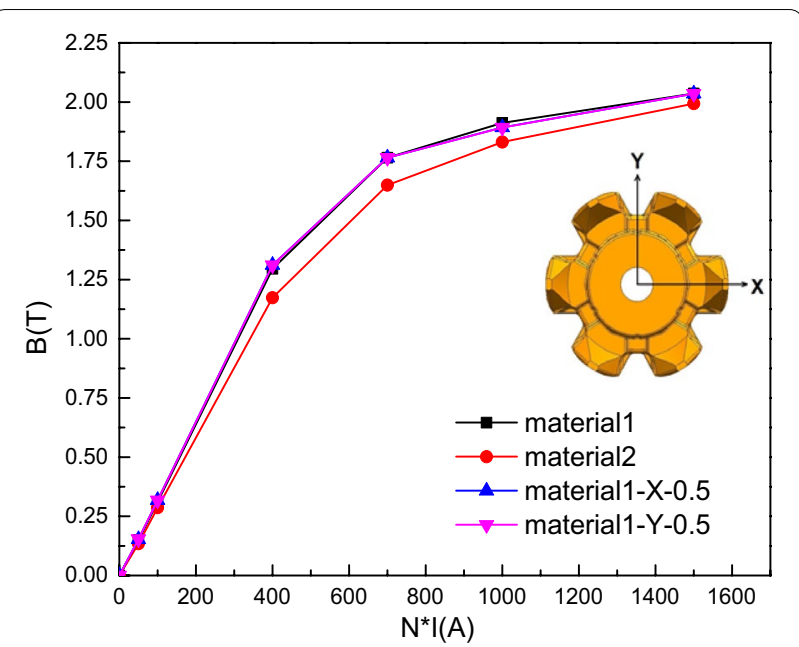

Figure 7 Magnetization-like curves obtained from simulation results

are also illustrated in Figure 7. The measured magnetization-like curves after offset were almost identical to the original curve. There were only subtle differences in individual points: this showed that the positioning error in the measuring process had almost no effect on the results. Therefore, the proposed NDTEM has good stability.

\section{Experimental Work}

To further study the relevant characteristics of the NDTEM, a group of claw poles were prepared for magnetic testing. As listed in Table 2, five materials, 06\#, $08 \#, 10 \#, 20 \#$, and DT4, were selected as the raw materials of the claw poles. The corresponding chemical compositions were tested and recorded. Cylinder rods with a diameter of $50.0 \mathrm{~mm}$ and height of $73.0 \mathrm{~mm}$ were heated up to $1200{ }^{\circ} \mathrm{C}$ by induction heating to undertake the hot-forging steps. After hot forging, three different heat-treatment strategies were adopted. Strategy I: The forgings were reheated to $950{ }^{\circ} \mathrm{C}$ and held for $3 \mathrm{~h}$, then cooled down in the furnace to a discharge temperature of $80{ }^{\circ} \mathrm{C}$. Strategy II: The residual heat of the forgings was used. The hot forgings were held in an insulated box that allowed the temperature to slowly decrease to $400{ }^{\circ} \mathrm{C}$ within $7 \mathrm{~h}$, and were then cooled to room temperature in air. Strategy III: The hot forgings, with a residual temperature around $900{ }^{\circ} \mathrm{C}$, were cooled to $400{ }^{\circ} \mathrm{C}$ within $10 \mathrm{~min}$ through a temperature-controlled mesh-belt furnace, and then cooled to room temperature in air. After heat treatment, a shot-blasting process was used to clear the surface scale and cold sizing was carried out to improve the accuracy of some local dimensions. Finally, three claw poles of the same dimensions made of $08 \#$ steel subjected to three different heat-treatment processes and 
Table 2 Chemical compositions of five claw pole materials

\begin{tabular}{|c|c|c|c|c|c|c|}
\hline Steel type & $C(\%)$ & $\mathrm{Si}(\%)$ & $M n(\%)$ & $\mathrm{P}(\%)$ & $\mathrm{S}(\%)$ & $\mathrm{Cr}(\%)$ \\
\hline 08\# & 0.069 & 0.240 & 0.385 & 0.017 & 0.009 & 0.008 \\
\hline 06\# & 0.054 & 0.092 & 0.319 & 0.015 & 0.002 & 0.003 \\
\hline $10 \#$ & 0.095 & 0.271 & 0.436 & 0.018 & 0.007 & 0.007 \\
\hline $20 \#$ & 0.180 & 0.300 & 0.450 & 0.020 & 0.015 & 0.010 \\
\hline DT4 & 0.004 & 0.072 & 0.060 & 0.014 & 0.008 & 0.002 \\
\hline
\end{tabular}

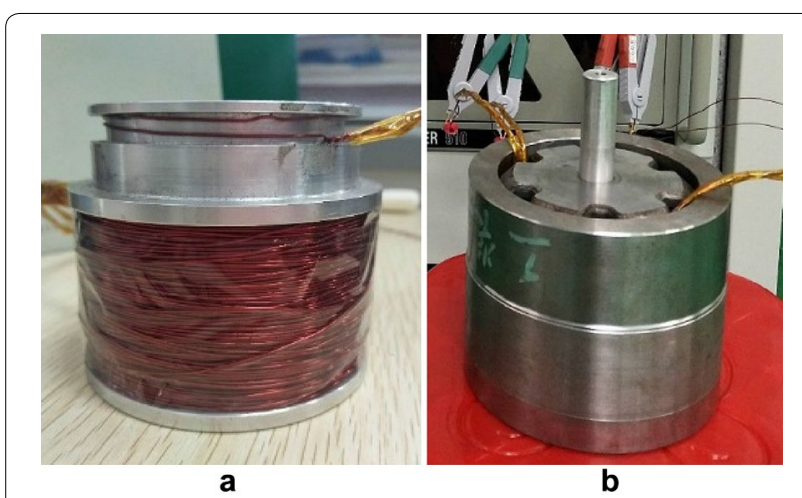

Figure 8 Measurement setup of the non-destructive test for the evaluation of magnetic properties (NDTEM) method: a Winding ferrule with magnetizing winding and search coil. b Assembly with measured claw pole

four other materials with the first heat-treatment strategy were obtained, giving a total of seven claw pole types.

\subsection{Evaluation of NDTEM}

To perform magnetic testing with the NDTEM, 400 turns of copper wire were uniformly wound layer-bylayer in the same direction onto the lower space of the winding ferrule to work as the magnetizing winding, and two turns of wire were wound on the upper space as the search coil, as shown in Figure 8a. The winding ferrule with wound coils was placed into the support base and the claw pole to be measured was installed on the support base through the positioning axis. The guide ring was then placed on the support base and the assembly was prepared for measurement as shown in Figure 8b. In the mass production, the outer diameter of the claw poles varies in a certain tolerance range. The designed maximum unilateral gap between the guide ring and claw pole is $0.5 \mathrm{~mm}$, and the positioning axis can ensure the uniform gap the inner hole of the claw is used as the reference for the turning of the outer dimeter. In the next step, the two poles of the magnetizing winding were connected to the positive and negative poles of a DC excitation current, and the search coil was connected to a fluxmeter. Finally, magnetization-like curves of each claw

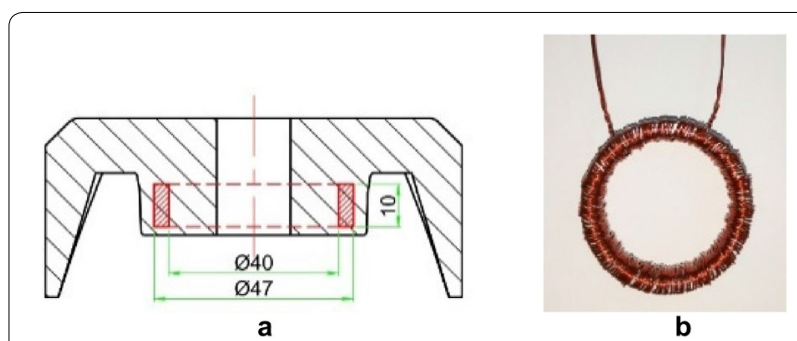

Figure 9 a Sampling position and $\mathbf{b}$ wound coils of ring sample

pole could be obtained. For each type of claw pole, five claw poles were each measured three times.

\subsection{Testing with Ring Sample}

For comparative analysis, a typical ring-sample method was also selected to evaluate the magnetic properties. After measuring the overall magnetic properties of the claw poles with the NDTEM, ring samples with a rectangular cross-section, where the inner diameter, outer diameter, and height were $40.0 \mathrm{~mm}, 47.0 \mathrm{~mm}$, and $10.0 \mathrm{~mm}$, respectively, were machined from the inner boss of the measured claw poles, as shown in Figure 9a. The surface roughness after machining was $R_{\mathrm{a}} 1.6 \mu \mathrm{m}$. To eliminate the influence of residual stress from machining, a $400{ }^{\circ} \mathrm{C}$ annealing process was carried out for all ring samples. Of course, the claw poles were destroyed during this preparation process. Before testing, a thin layer of electrically insulating tape was used to cover the surface of the ring sample. Twenty turns of search coils were wound and another insulating layer was placed, then 150 turns of magnetizing coils were wound. The ring sample with wound coils, shown in Figure 9b, could then be measured and the magnetization curve of each claw pole obtained.

\section{Results and Discussion}

\subsection{Consistency Evaluation}

The magnetization-like curves of the seven claw pole types measured by the NDTEM and the corresponding magnetization curves measured by the ring-sample method are shown in Figures 10 and 11, respectively. 

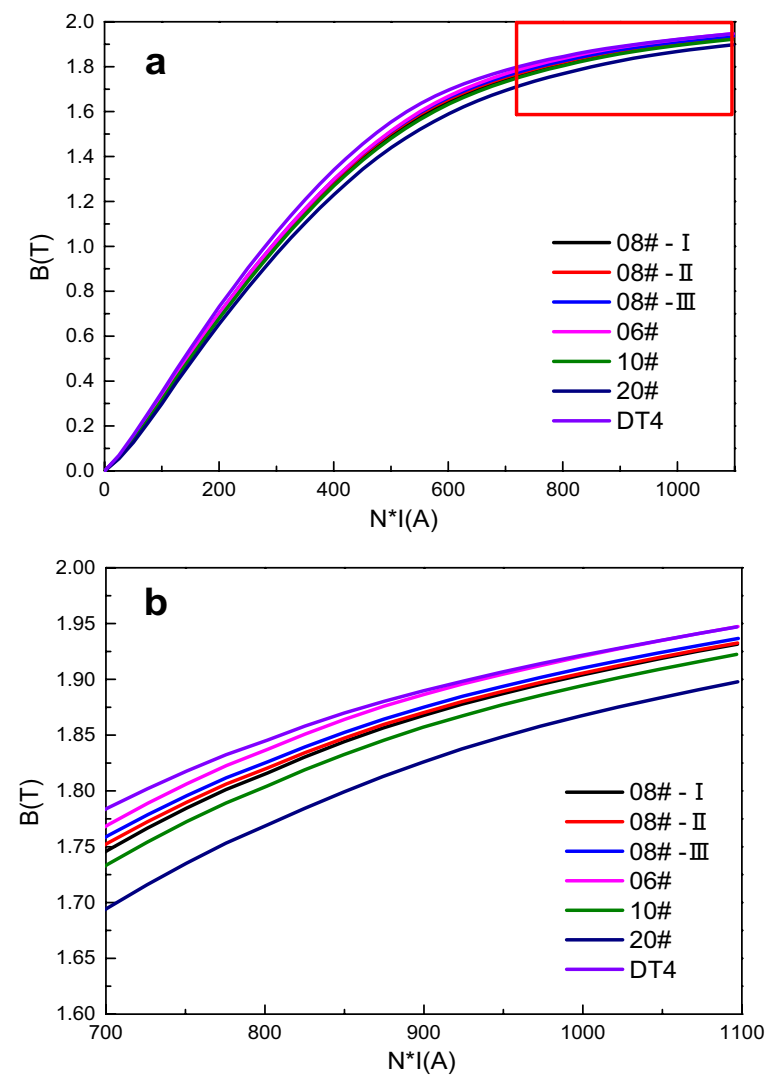

Figure $\mathbf{1 0}$ a Magnetization-like curves and $\mathbf{b}$ partially enlarged view at 700-1100 A of results of the non-destructive test for evaluation of magnetic properties (NDTEM)

The magnetic induction intensity increased with the excitation current. The increased amplitude was relatively large in the initial phase, then tended to saturate under large excitation currents. When partially enlarged views were observed, the trend of measured curves from the NDTEM were always consistent, as shown in Figure 10b; however, a crossover phenomenon of the curves measured by the ring-sample method occurred. When the excitation magnetic field was less than $2500 \mathrm{~A} / \mathrm{m}$, the curve of DT4 was the highest; when the excitation magnetic field exceeded $2500 \mathrm{~A} / \mathrm{m}$, the curve of $06 \#$ became the highest. Moreover, when the excitation magnetic field strength reached $3500 \mathrm{~A} / \mathrm{m}$, the magnetization curves of 08\#-II and 08\#-III, 08\#, and 10\# appeared to intersect, or even locally coincide, as shown in Figure 11b. It was therefore very difficult to choose the claw pole with the best magnetic property based on these confusing results of the ring-sample method. The behavior of the different claw poles could, however, be distinguished from the results of the NDTEM.

From the above comparison analysis, it was inferred that the NDTEM for claw poles showed better
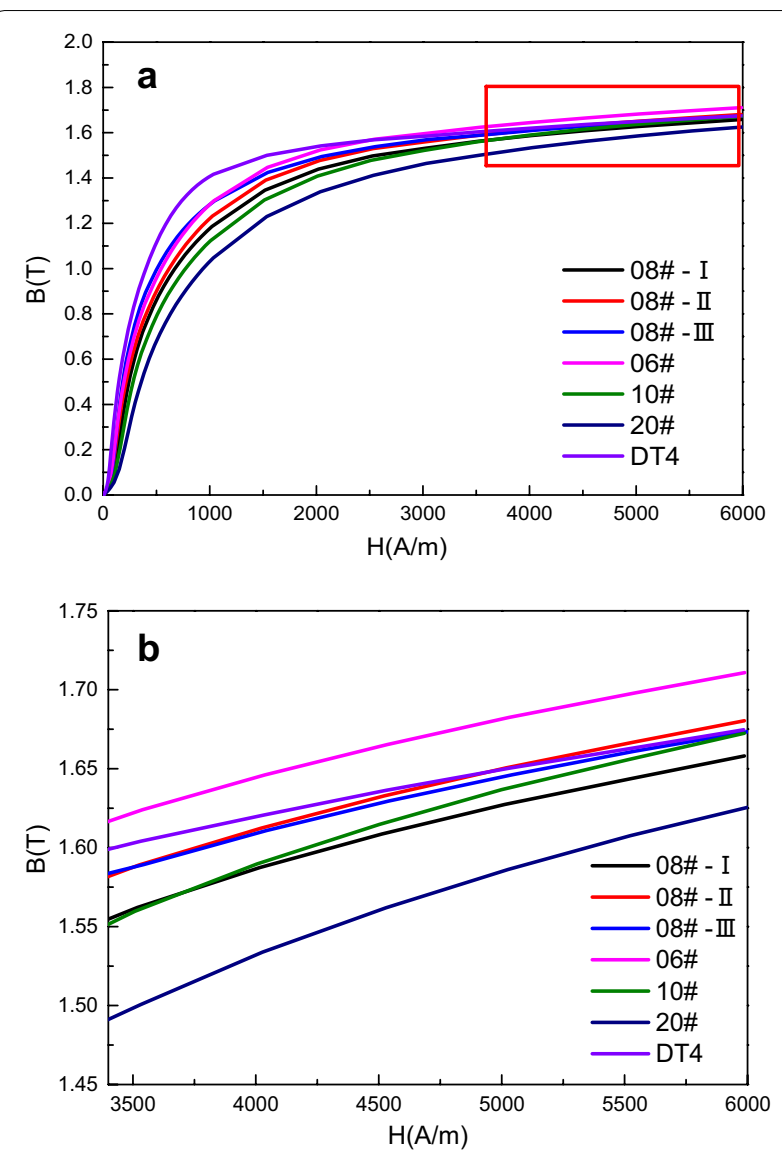

Figure 11 a Magnetization curves and $\mathbf{b}$ partially enlarged view at $3400-6000 \mathrm{~A} / \mathrm{m}$ of results of the ring-sample method

consistency than the ring-sample method. The main reason is that the machining and subsequent heat-treatment processes used for preparing the ring samples introduced some degree of error. Furthermore, a ring sample taken from a claw pole may only reflect the magnetic property of the boss, but the NDTEM represented the overall magnetic properties of the claw pole.

\subsection{Accuracy Analysis}

To analyze the accuracy of the two methods, the measured magnetic induction intensities at the initial and saturation stages of different types of claw poles were extracted. As shown in Figure 12, the measured values were below $500 \mathrm{~A}$ in the initial phase and $1100 \mathrm{~A}$ at the saturation stage using the NDTEM and below $2000 \mathrm{~A} / \mathrm{m}$ in the initial phase and $6000 \mathrm{~A} / \mathrm{m}$ at the saturation stage by the ring-sample method, with each claw pole tested three times. In general, the measurement error of these two methods was very small: the maximum error was less than $0.01 \mathrm{~T}$ and the maximum absolute error was 

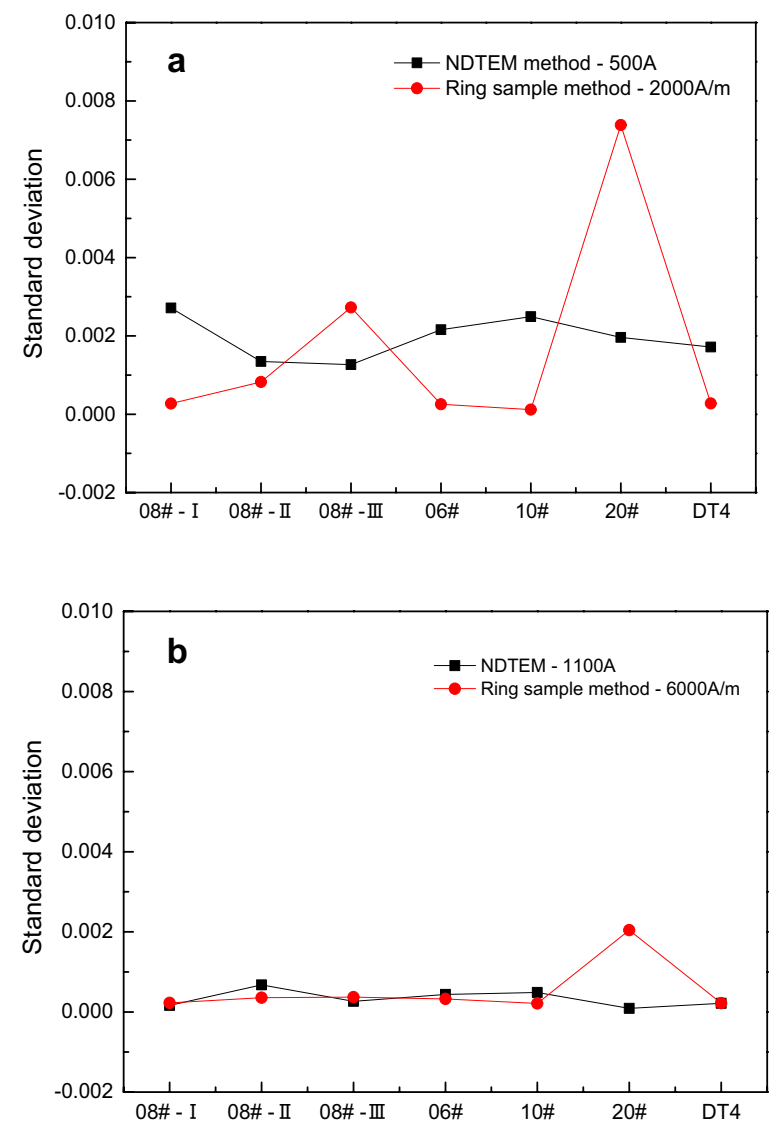

Figure 12 Standard deviation values at a initial stage and $\mathbf{b}$ saturation stage for the two methods

less than 6\%. Therefore, the measuring accuracy of both methods was relatively high and acceptable for industry.

When comparing the results of the initial and saturation stages, the measured errors of the latter were relatively small because the change of the magnetic property itself was not large. When the measured values of the initial stage were checked in detail from Figure 12a, the specific measured errors of the seven different types of claw poles varied from $0.0001 \mathrm{~T}$ to $0.0074 \mathrm{~T}$ when using the ring-sample method, while the errors of the NDTEM method only changed from $0.0013 \mathrm{~T}$ to $0.0027 \mathrm{~T}$. From this point of view, the applicability of the proposed NDTEM is better than that of the conventional ring-sample method.

\subsection{Stability Analysis}

To check the stability, five samples of each type of claw pole were measured. The measured values at the initial and saturation stages of the two methods are presented in Figure 13. In the initial stage, the deviation in values determined by the NDTEM varied from $0.0019 \mathrm{~T}$ to $0.0080 \mathrm{~T}$, but this increased to range from $0.0075 \mathrm{~T}$ to $0.0165 \mathrm{~T}$ when using the ring-sample method. In the saturation stage, the measurement errors varied from $0.0094 \mathrm{~T}$ to $0.0159 \mathrm{~T}$ for the ring-sample method, while those of the NDTEM remained within the range of $0.0004 \mathrm{~T}$ to $0.0031 \mathrm{~T}$. In general, the absolute relative error of the NDTEM was only $5.3 \%$. Therefore, the stability and repeatability of the NDTEM method could be guaranteed and were better than those of the ring-sample method.

The relative difference $(R D)$ between the measured magnetic induction intensities of different claw poles was calculated using Eq. (3):

$$
R D=\frac{\text { Max }- \text { Min }}{\text { Mean }}
$$

where Max, Min, and Mean designate the maximum, minimum, and average values of the measured magnetic induction intensity of different claw poles under the same excitation condition, respectively.

As seen from Table 3, the $R D$ values of the NDTEM were $7.49 \%$ and $2.60 \%$ for the two different stages, which are lower than those of the ring-sample method. This indicates that the NDTEM method is less sensitive to changes in magnetic properties of claw poles. Therefore, improvement in the sensitivity of the new method should be paid more attention in the next phase of research.

\subsection{Evaluation of Magnetic Properties of Different Claw Poles}

As discussed above, the consistency, accuracy, and stability of the NDTEM were better than those of the ringsample method. In this case, the measured results from the NDTEM were used to evaluate the magnetic properties of different claw poles.

From Figures 13a and b, the measured magnetic induction intensities of claw poles made from five different materials treated using the first heat-treatment strategy showed that the claw pole made of DT4 had the best magnetic performance and that made of $20 \#$ steel was the worst. Considering the chemical composition of the materials (Table 1), it was found that the higher the carbon content, the worse were the magnetic properties. The main reason is the pinning effect of carbide on magnetic domains [26].

When claw poles made of the same material (08\# steel) were checked, the magnetic induction intensities differed for the three different heat-treatment processes: the corresponding order was $08 \#-\mathrm{I}<08 \#-\mathrm{II}<08 \#$-III. The faster the cooling rate and the higher the discharge temperature, the better were the magnetic properties. The results can be explained from the effects of cooling rate and discharge temperature on grain size and carbide distribution: the faster the cooling rate, the lower the grain 

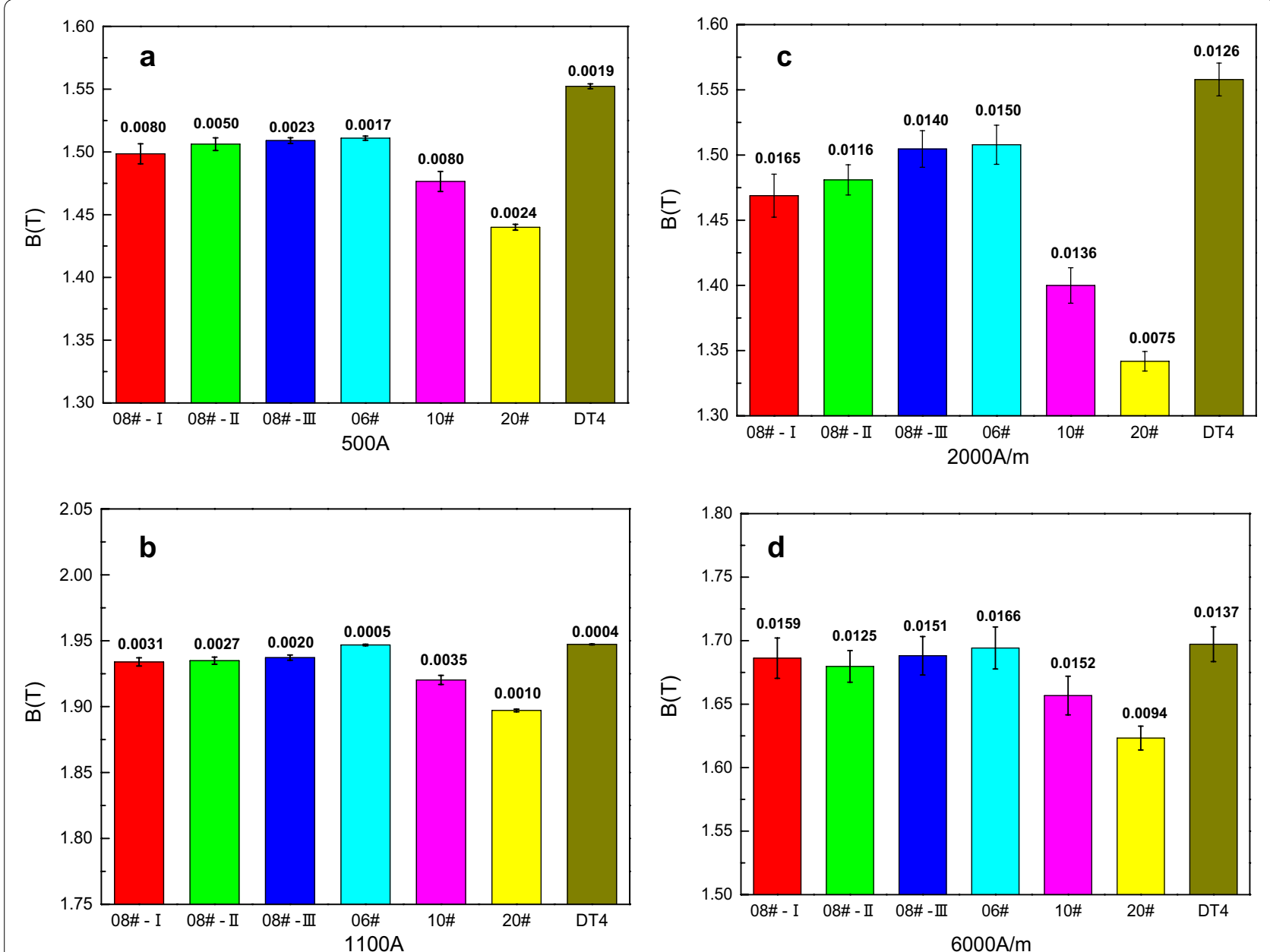

Figure 13 Magnetization values of five samples at $\mathbf{a} 500 \mathrm{~A}$ and $\mathbf{b} 1100 \mathrm{~A}$ measured by the non-destructive test for evaluation of magnetic properties (NDTEM) and at c $2000 \mathrm{~A} / \mathrm{m}$ and $\mathbf{d} 6000 \mathrm{~A} / \mathrm{m}$ measured by the ring-sample method

Table 3 Relative differences in measured magnetic induction intensities of different claw poles by the two methods (\%)

\begin{tabular}{|c|c|c|c|c|}
\hline & \multicolumn{2}{|c|}{ NDTEM method } & \multicolumn{2}{|c|}{ Ring sample method } \\
\hline & $500 \mathrm{~A}$ & $1100 \mathrm{~A}$ & $2000 \mathrm{~A} / \mathrm{m}$ & $6000 \mathrm{~A} / \mathrm{m}$ \\
\hline$R D$ & 7.49 & 2.60 & 14.74 & 4.41 \\
\hline
\end{tabular}

growth and, although fine grains can increase coercivity, the carbide distribution was more uniform. A fast cooling rate meant that the time for carbide precipitation and aggregation at the grain boundary was short, and thus improved the magnetic properties. The higher the discharge temperature, the shorter the cooling time in the furnace, which also inhibited precipitation of carbide. The effect of carbon content on the magnetic properties of the forged claw pole was much greater than that of the heat-treatment process parameters. The carbon content of low-carbon steel is the most important factor affecting the magnetic properties of materials [27].

\section{Conclusions}

(1) A non-destructive testing method for evaluating the magnetic properties of claw pole was proposed and a corresponding measurement set-up designed. The designed magnetic circuit has a high degree of similarity with the magnetic circuit formed by a claw pole working in a motor. The excitation effect of the magnetizing winding is the same as that of the magnetizing winding inside the rotor of a claw pole generator. Magnetization-like curves of magnetic induction intensity that varied with the excitation current for the claw pole were measured.

(2) An equivalent static magnetic analysis model was created to simulate the magnetic measurement pro- 
cess of the NDTEM. FE simulation results showed that the curves obtained from the NDTEM system had good agreement with the trend of the input magnetic curves, and the magnetization-like curve could reflect and identify the magnetic performance of the claw pole. A significant finding was that the positioning error of the claw pole during measurement hardly affected the measured results.

(3) To validate the NDTEM, a group of claw poles, made of five different materials (06\#, 08\#, 10\#, $20 \#$, and DT4) and treated by three different heattreatment processes, were prepared for magnetic testing. All the claw poles were measured by the NDTEM and a typical ring-sample methods. The results proved that the NDTEM had better consistency, relatively higher accuracy, and much stronger stability.

(4) A preliminary study on the effect of material components and manufacturing processes on the magnetic properties was carried out. The higher the carbon content, the worse the magnetic properties of the claw pole were. A larger cooling rate and higher discharge temperature were helpful to improve the magnetic properties of the claw poles.

(5) However, the sensitivity of the NDTEM method is lower than that of the ring-sample method. In future, the sensitivity needs to be improved, and the gap between the guide ring and claw pole as key impactor should be investigated in detail

\section{Authors' Contributions}

$\mathrm{ZZ}$ and $\mathrm{CH}$ were in charge of the whole trial; $\mathrm{CH}$ and $\mathrm{XB}$ wrote the manuscript; MT assisted with the experiment; XT assisted with technique check. All authors read and approved the final manuscript.

\section{Authors' Information}

Chengliang Hu, born in 1980, is currently a professor at Shanghai Jiao Tong University, China.

Xuejiao Bai, born in 1996, is currently a master candidate at Shanghai Jiao Tong University, China.

Minjun Tang, born in 1980, is currently an engineer at Jiangsu Longcheng Precision Forging Co., Ltd., China.

Xiaofeng Tang, born in 1973, is currently a senior engineer at Jiangsu Longcheng Precision Forging Co., Ltd., China.

Zhen Zhao, born in 1972, is currently a professor at Shanghai Jiao Tong University, China.

\section{Competing Interests}

The authors declare that they have no competing interests.

\section{Funding}

Partially supported by National Natural Science Foundation of China (Grant No. 51875348)

\footnotetext{
Author Details

${ }^{1}$ Institute of Forming Technology \& Equipment, School of Material Science and Engineering, Shanghai Jiao Tong University, Shanghai 200030, China.

2 Jiangsu Longcheng Precision Forging Co., Ltd., Jiangsu 213100, China.
}

Received: 26 April 2019 Revised: 23 September 2019 Accepted: 24 September 2019

Published online: 23 October 2019

\section{References}

[1] F N Jurca, C Martis. Theoretical and experimental analysis of a threephase permanent magnet claw-pole synchronous generator. IET Electric Power Applications, 2012, 6: 491-503.

[2] E Doege, R Bohnsack. Closed die technologies for hot forging. Journal of Materials Processing Technology, 2000, 98: 165-170.

[3] CYang, S D Zhao, J J Zhang. A single stage hot forging process and die set of alternator poles. International Journal of Material Forming, 2013, 6: 511-517.

[4] X R Xin, D Xu, C S He, et al. Claw-pole closed forging process. Advanced Materials Research, 2014, 912-914: 605-608.

[5] CYang, S D Zhao, J J Zhang. A combined radial forging-forward extrusion forming process of alternator poles. Journal of Materials Engineering and Performance, 2014, 23: 108-114.

[6] Q C Li, L Ma, J Chen, et al. Influence of claw pole forging procedure on power performance of automobile generator. Hot Working Technology, 2015, 44(23): 42-45. (in Chinese)

[7] J Pal'a, O Stupakov, J Bydžovský, et al. Magnetic behaviour of low-carbon steel in parallel and perpendicular directions to tensile deformation. Journal of Magnetism and Magnetic Materials, 2007, 310: 57-62.

[8] B D Cullity, C D Graham. Chapter 2: Experimental methods. In: Introduction to magnetic materials. 2nd ed. IEEE Press, New Jersey, USA, 2009.

[9] J H Li, C Yuan, W L Zhang, et al. Effects of precipitate element addition on microstructure and magnetic properties in magnetostrictive Fe83Ga17 alloy. Journal of Magnetics, 2016, 21: 12-19.

[10] TInoue, KYamauchi, F Fujita. Effects of alloying element balance and micro-alloying elements on magnetic properties and hot ductility of PC permalloy. Materials Transactions, 2008, 49: 650-654.

[11] International Standard: IEC-60404-4 Magnetic Materials - Part 4: Methods of measurement of d.c. magnetic properties of magnetically soft materials. 2008.

[12] ZY Chen. Research on the effects of composition and structure on the magnetic of claw pole steel. Liaoning University of Technology, 2015. (in Chinese)

[13] M Pérez, R Ranchal, I de Mendizábal Vázquez, et al. Combined alternating gradient force magnetometer and susceptometer system. Review of Scientific Instruments, 2015, 86(1): 015110.

[14] M Kuru, E Ongun, A Özmetin, et al. Fabrication and characterization of permanent magnetic SmCo5 thin films by SQUID magnetometer. Materials Science Forum, 2018, 915: 16-21.

[15] J H Storm, P Hömmen, D Drung, et al. An ultra-sensitive and wideband magnetometer based on a superconducting quantum interference device. Applied Physics Letters, 2017, 110(7): 072603.

[16] X Lin, H S Liu, LT Dou, et al. Soft magnetic properties and microstructure of Fe84-xNb2B14Cux nanocrystalline alloys. Materials and Design, 2014, 56: 227-231.

[17] B Aslibeiki. Magnetic interactions and hysteresis loops study of Co/ CoFe2O4 nanoparticles. Ceramics International, 2016, 42: 6413-6421.

[18] T M El-Alaily, M K El-Nimr, S A Saafan, et al. Construction and calibration of a low cost and fully automated vibrating sample magnetometer. Journal of Magnetism and Magnetic Materials, 2015, 386: 25-30.

[19] G P Lin, P C Kuo, P L Lin, et al. Magnetic properties and microstructure of $\mathrm{TbCo} /(\mathrm{SiNx} / \mathrm{Co}) \mathrm{n}$ films. Materials and Design, 2010, 31: 1734-1736.

[20] M S Boon, W P Serena Saw, M Mariatti. Magnetic, dielectric and thermal stability of Ni-Zn ferrite-epoxy composite thin films for electronic applications. Journal of Magnetism and Magnetic Materials, 2012, 324: 755-760.

[21] D Jordán, D González-Chávez, D Laura, et al. Detection of magnetic moment in thin films with a home-made vibrating sample magnetometer. Journal of Magnetism and Magnetic Materials, 2018, 456: 56-61.

[22] R N Faria, A J Williams, I R Harris. Permeameter measurements of anisotropic PrFeCoBZr hydrogenation disproportionation desorption and recombination (HDDR) magnets. Journal of Magnetism and Magnetic Materials, 1999, 202: 349-353. 
[23] SY Shin, K H Shin, J S Kim, et al. High frequency permeameter with semirigid pick-up coil. Journal of Magnetism and Magnetic Materials, 2006, 304: e480-e482.

[24] P Anderson. A universal DC characterisation system for hard and soft magnetic materials. Journal of Magnetism and Magnetic Materials, 2008, 320: e589-e593.

[25] D X Chen, Y H Zhu, L X Xiang, et al. Calibration of a permeameter for measuring soft magnetic materials using long cylindrical samples characterized by demagnetizing-corrected solenoid method. Journal of Magnetism and Magnetic Materials, 2018, 458: 137-146.
[26] D C Jiles, D L Atherton. Theory of the magnetization process in ferromagnets and its application to the magnetomechanical effect. Journal of Physics D: Applied Physics, 1984, 17: 1265-1281.

[27] R Ranjan, D C Jiles, P K Rastogi. Magnetic properties of decarburized steels: an investigation of the effects of grain size and carbon content. IEEE Transactions on Magnetics, 1987, 23: 1869-1876.

\section{Submit your manuscript to a SpringerOpen ${ }^{\circ}$ journal and benefit from:}

- Convenient online submission

- Rigorous peer review

- Open access: articles freely available online

- High visibility within the field

- Retaining the copyright to your article

Submit your next manuscript at $\boldsymbol{\nabla}$ springeropen.com 